C-A/AP/\#138

February 2004

\title{
1.5-GeV FFAG Accelerator for the AGS Facility
}

A.G. Ruggiero, M. Blaskiewicz, E. Courant, D. Trbojevic, N. Tsoupas, W. Zhang

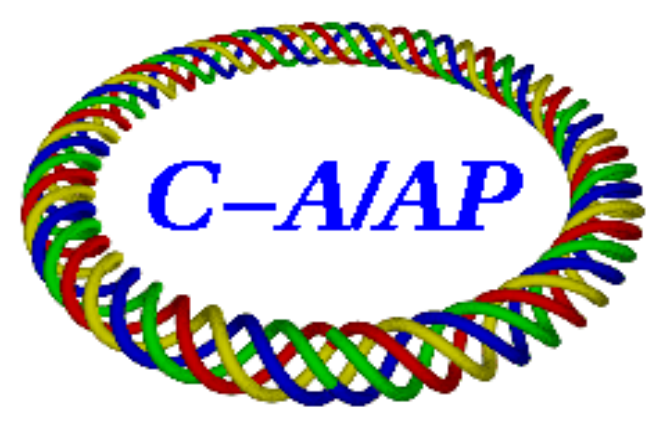

Collider-Accelerator Department Brookhaven National Laboratory Upton, NY 11973 


\title{
1.5-GeV FFAG Accelerator for the AGS Facility*
}

\author{
A.G. Ruggiero, M. Blaskiewicz, E. Courant, D. Trbojevic, N. Tsoupas, W. Zhang \\ Brookhaven National Laboratory \\ February 2004
}

\section{Abstract}

In this report we propose that a $1.5-\mathrm{GeV}$ FFAG accelerator is considered as a substitute (replacement, if you wish) of the present 1.5-GeV AGS Booster. The substitution will enhance the performance of the AGS accelerator facility in a variety of ways. First of all, it would still allow preparation and acceleration of all hadronic types of particles, namely: protons, negative hydrogen ions, polarized protons and heavy ions. The major benefit is that it would considerably shorten the typical combined AGS acceleration cycle. As a consequence we expect an improvement of the beam stability and performance, an increase of beam intensity, and small beam dimensions. At the same time, the $1.5-\mathrm{GeV}$ FFAG accelerator is, in our opinion, a better alternative to the 1.2-GeV SCL that has been proposed for the AGS upgrade toward an average proton beam power of $1 \mathrm{MW}$. It is conceptually easier to design, it requires less of engineering involvement and, very important, is less costly to build and to operate. We also present a preliminary design of the FFAG accelerator that proves its feasibility. Basing on that, we propose that a more detailed design is undertaken.

\section{Introduction}

The concept of the Fixed-Field Alternating-Gradient (FFAG) accelerator was introduced in the middle of last century [1]. Thus this technology cannot be considered recent or new. Soon after the invention, several accelerators adopting the concept of fixed field where demonstrated, and made successfully operational. But all of them accelerated electron beams [2]. Acceleration of protons was proposed at different times subsequently, mostly in connection of high power neutron spallation sources [3], but none of these projects materialized. Only recently storage of protons in relatively low-energy (10-150 $\mathrm{MeV}$ ) FFAG accelerators was proven at KEK in Japan [4]. There is recently a revival of interest in this type of technology especially for the generation of proton beam power in the MW range, and energy of about one GeV or in that proximity. This technology, if successfully demonstrated, would represent a considerable shortcut at a more modest cost and human investment than the Super-Conducting Linacs that have in the meantime been proposed for the same scope [5].

At the other end of the spectrum, there is also renewed interest in the FFAG for acceleration of electrons [6] and muons [7]. For this type of particles, because they are ultra-relativistic, that is their velocity is already close to the light velocity, and it does not

\footnotetext{
* Work performed under the auspices of the U.S. Department of Energy
} 
change appreciably, acceleration is conceptually simpler when compared to protons. Because of their heavier mass, the velocity of protons changes considerably during the acceleration cycle. Condition for isochronous motion, that is required for effective acceleration, is difficult to be met in the case of protons (as well of polarized protons, negative hydrogen ions, and heavy ions). One thus needs some additional invention, like that described later, to make the use of the FFAG accelerator appealing also for the case of protons. The present report describes how a 1.5-GeV FFAG accelerator, as a replacement of the $1.5-\mathrm{GeV}$ AGS-Booster, could benefit the performance of the BNLAGS complex.

\section{The BNL-AGS Facility}

The BNL-AGS accelerator complex is shown schematically in Fig. 1. It is made of five different accelerators :

- The 200-MeV room-temperature Drift-Tube Linac (DTL) operating at 201.25 $\mathrm{MHz}$.

- The Heavy-Ion Tandems that provide the intense, short beam pulses of Heavy Ions. Depending on the particular mode of operation selected, this facility runs in parallel to the proton DTL.

- The AGS Booster for the acceleration of protons $\left(\mathrm{H}^{-}\right.$, and polarized protons) to $1.5 \mathrm{GeV}$, as well of heavier ions (stripped or partially stripped) to other intermediate energies.

- The Alternating-Gradient Synchrotron (AGS) that accelerates all type of particles to energy, for instance, of $28 \mathrm{GeV}$ in the case of protons, and $12 \mathrm{GeV} / \mathrm{u}$ for ions of $\mathrm{Au}$.

- Finally, whatever beam has been accelerated in the AGS it can either be sent toward an external fixed-target experimental area, or injected into the Relativistic Heavy-Ion Collider (RHIC) for farther acceleration to $250 \mathrm{GeV}$ for protons and $100 \mathrm{GeV} / \mathrm{u}$ for ions of gold, followed by storage and collision.

Thus the accelerator facility of Brookhaven National Laboratory (BNL), centered around the AGS, allows an all inclusive program of research with protons, negative hydrogen ions $\left(\mathrm{H}^{-}\right)$, polarized protons, and Heavy Ions from Deuterons up to ions of Gold and, in the future, possibly Uranium. It is obvious the significant role that is played by the AGS-Booster for the collection and preparation of these types of particle beams before they

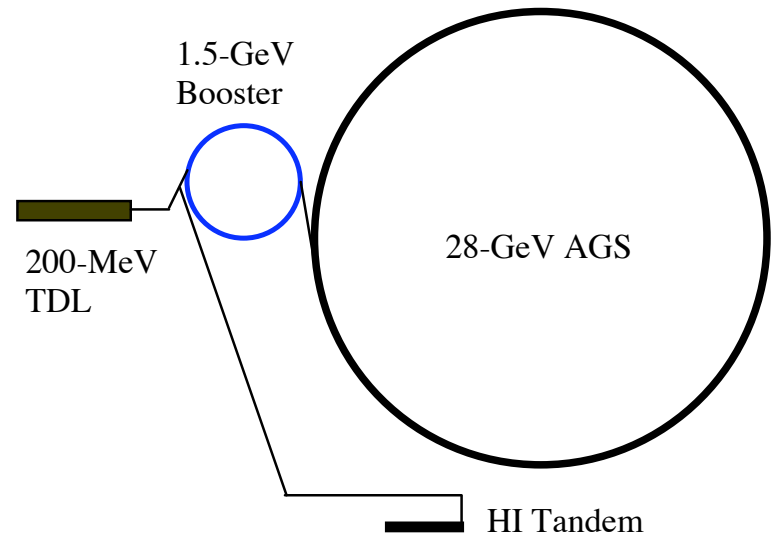

Figure 1. The AGS Accelerator Complex are transferred to the AGS for the final acceleration proper. Yet the Booster is a relatively slow-cycling accelerator, and several Booster cycles are required for an efficient filling of 
the AGS. For instance a typical mode of operation for protons is shown in Fig. 2. In this case, negative ions are injected into the Booster at $200 \mathrm{MeV}$, where they are stored by charge-exchange method. The emerging positive-charged protons are accelerated to 1.5 $\mathrm{GeV}$ and transferred to the AGS that is initially set in a holding mode. The AGS circumference is four times that of the Booster, so that a complete fill of the AGS requires also four Booster beam pulses. Once the AGS is completely filled, the whole beam is then accelerated to the top energy of about $28 \mathrm{GeV}$. Presently, the repetition rate of the Booster is $7.5 \mathrm{~Hz}$, and that of the AGS is $0.5 \mathrm{~Hz}$. That requires a filling time of the AGS of about 0.5 second, followed by about one second for acceleration to the top energy, and another second for resetting the AGS field cycle. At best, the overall cycle period is 2.5 seconds (excluding any flat-top for slow-spill extraction, namely only single-turn extraction). With a typical intensity of $7 \times 10^{13}$ protons per cycle accelerated to $28 \mathrm{GeV}$, that yields an average proton beam power of about $125 \mathrm{~kW}$, in the best of conditions.

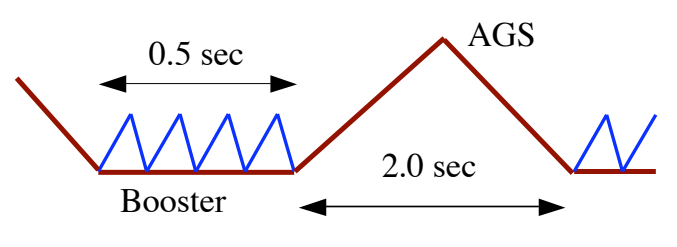

Figure 2. Typical AGS cycle for protons

It is also to be noticed that a filling time of 0.5 second is actually a long period where several effects on the stored beam may occur, with consequent losses and size deterioration. Similar considerations apply of course also to polarized protons and heavy ions. One would expect a great improvement on the beam quality and performance if the filling time can be shortened so that particles do not have to wait too long before they are accelerated.

\section{The AGS Upgrade Program}

It has been recently proposed [8] to upgrade the AGS facility to generate an average proton beam power of at least $1 \mathrm{MW}$. This can be accomplished in two steps: (1) the replacement of the main magnet AGS power supply with one operating at $2.5 \mathrm{~Hz}$, and (2) a Super-Conducting Linac (SCL) for direct acceleration of protons (actually $\mathrm{H}^{-}$) from the 200-MeV DTL to $1.2 \mathrm{GeV}$, followed by immediate transfer into the AGS, as shown in Fig. 3. This mode of operation would completely by-pass the Booster, and eliminate the $0.5 \mathrm{~s}$ long injection period. The proposed new AGS cycle is then as shown in Fig. 4. With this done, it is also reasonable to expect a significant improvement on the beam performance (namely, stability and dimensions confinement), and a 30 percent increase in overall intensity per AGS cycle, that is about $10^{14}$ protons (with some contingency included). Said in another way, the shorter is the period of time the beam spends circulating in the Booster or in the AGS, the more stable it is, and it can carry more intensity.

A feasibility study of the 1.2-GeV SCL has already been done [9], and more engineering design is under progress. Such a device is relatively expensive, and is also a technology that still needs to be completely demonstrated (see for instance the SNS-SCL [10]). Moreover, it requires an extensive men power and engineering knowledge in cryogenic and high-gradient RF cavities. In parallel, the upgrade requirement of the main magnet AGS power supply had been estimated, and a conceptual design of the new power supply 
layout already carried out [11]. Though the power supply upgrade can eventually result in a quite costly item, nevertheless it is perceived as relatively straightforward, without conceptual difficulties. Yet, this upgrade program is useful exclusively to the case of protons. Heavy Ions still need the Booster for the intermediate steps of acceleration.

On the other end, a $1.5-\mathrm{GeV}$ FFAG accelerator represents a valid alternative to the $1.2-\mathrm{GeV}$ SCL, fulfilling the same power requirements and much more. In this note we explore its possible use for the AGS facility. It is a summary of our initial feasibility study. We propose that it is given a closer consideration for farther studies. After all, before BNL can commit to either the SCL or the FFAG alternative, there is still plenty of time.

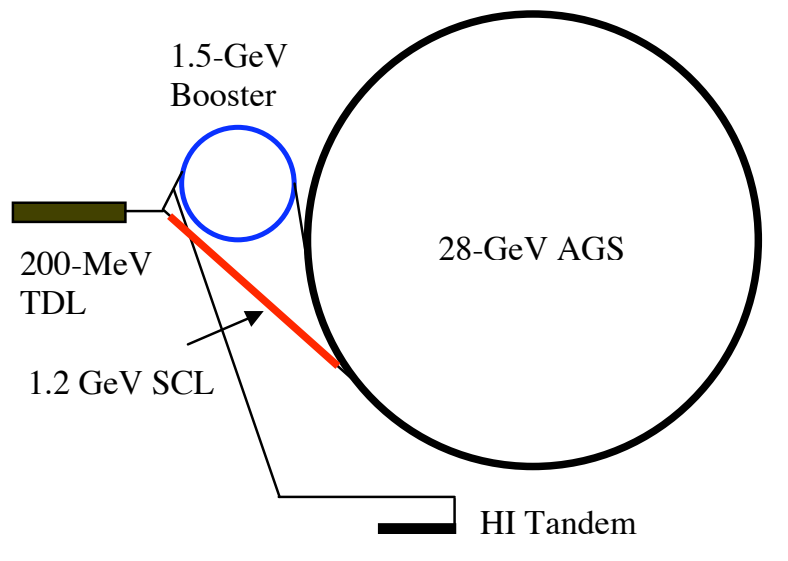

Figure 3. AGS Upgrade with $1.2 \mathrm{GeV}$ SCL

\section{Advantages of the FFAG accelerator}

The bending and focusing magnet fields are kept constant during acceleration. The main power supply is thus continuous. The magnets are not ramped, either way, and the resulting cycle can be made considerably shorter, just what is required for acceleration from $200 \mathrm{MeV}$ to $1.5 \mathrm{GeV}$. Assuming the FFAG ring has the same size of the present Booster, an energy gain of $1 \mathrm{MV} /$ turn requires a total acceleration cycle of only one millisecond. Assuming again that four FFAG cycles are needed for the filling of the AGS, the filling time does not exceed 4-5 msec. Unfortunately, the 200-MeV DTL cannot be operated, without major modifications, for a period of time longer than $1 \mathrm{msec}$ at $2.5 \mathrm{~Hz}$ repetition rate. We shall show later an acceleration scheme based on the harmonic jump that may require no more than $1 \mathrm{msec}$ for the total AGS filling time.

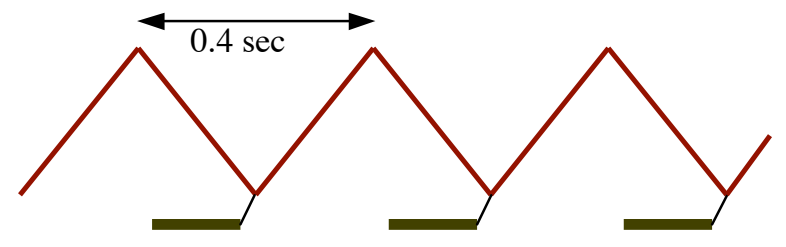

Figure 4. AGS Cycle with 1.2-GeV SCL or 1.5-GeV FFAG

The FFAG can be made compact, just about the same size of the Booster, and be installed in the same Booster enclosure, with and without the Booster itself. Or, alternatively, it can be lodged in a separate enclosure located more or less at the location of the proposed 1.2-GeV SCL, as shown in Fig. 5. The FFAG is essentially made of conventional, relatively low field magnets. Superconducting technology is not required, removing the need of cryogenics, and the chemical processing otherwise required for RF cavities. The level of engineering expertise needed is thus minimum, with less investment of men power, at a cost that can be only a small fraction of the $1.2-\mathrm{GeV}$ SCL. 
Differently from the SCL, the FFAG can accelerate all types of particles; protons, negative hydrogen ions, polarized protons, and Heavy Ions. Each beam will spend only a very small short period of time for acceleration, and will thus be less exposed to damaging effects of depolarization, stripping, losses, and size growth. For instance the vacuum requirements are relaxed. Also, considering that the beam spends at most a hundred revolutions between injection and extraction, magnet imperfections and other errors may not have the time to impact significantly on the beam dynamics.

The proton beam is still provided by the 200-MeV DTL. At injection the beam is bunched at 201. $25 \mathrm{MHz}$. At that frequency, the RF bunch dimensions are very small, both in length and in the momentum spread. This is a feature that we like to preserve by avoiding dilution when filling lower frequency RF buckets. In the FFAG it is possible to accelerate protons at the crest (that is at $90^{\circ}$ ) of the $201.25-\mathrm{MHz}$ RF waveform. Nevertheless, as we have already

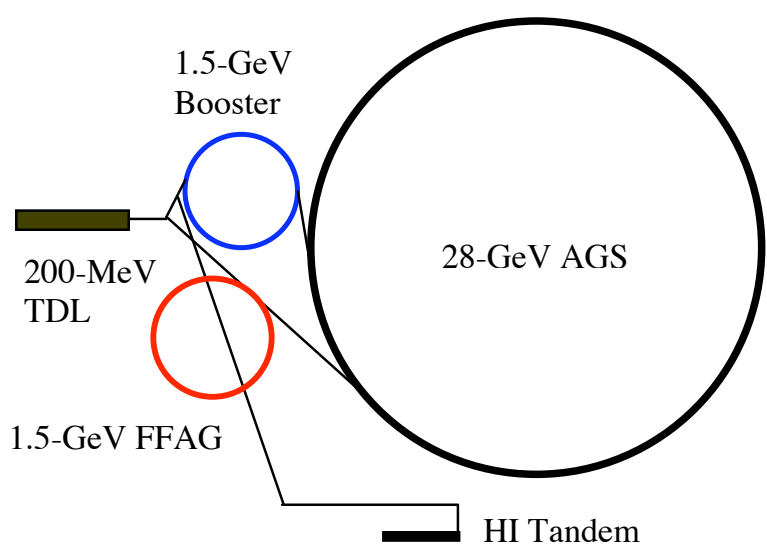

Figure 5. AGS Upgrade with 1.5-GeV FFAG hinted in the introduction, the proton beam velocity varies considerably between $200 \mathrm{MeV}$ and $1.5 \mathrm{GeV}$, thus the accelerating RF voltage will have to be modulated in frequency over the range between 200 and 320 MHz. Such modulation over a period of one millisecond, accompanied by phase stabilization, is very difficult, if not impossible. Thus we need to keep the RF constant and modulate the voltage in the manner we shall describe later.

To provide the required intensity in the AGS, each FFAG pulse carries $2.5 \times 10^{13}$ protons, somewhat larger than what the present Booster can deliver. The required average RF power is then $50 \mathrm{~kW}$. But the duty cycle is now (4 cycles) $\mathrm{x}(1 / 4 \mathrm{~ms}) \times(2.5 \mathrm{~Hz})=0.25 \%$, so that the RF peak power required during the acceleration cycle in the FFAG is $20 \mathrm{MW}$. This may be a large value, comparable to that of the 1.2-GeV SCL design where the duty cycle was also $0.2 \%$. Thus, it is important that the issue of beam loading of the RF cavities system is addressed.

It is reasonable to assume that the full intensity of $2.5 \times 10^{13}$ protons per FFAG pulse can be attained with multi-turn injection by charge exchange as it is presently done in the Booster. We shall assume that modifications of the DTL, and in particular of the frontend combination of the ion source and RFQ, can lead to a beam improvement with a delivered intensity at $200 \mathrm{MeV}$ of $30 \mathrm{~mA}$ within the Linac pulse, and a smaller normalized emittance of $1 \pi \mathrm{mm}-\mathrm{mrad}$ (rms). To get then the required number of protons per FFAG pulse, one needs a Linac pulse length of $135 \mu \mathrm{s}$. This should be within the performance capability of the DTL, except that now 4 pulses each of that duration are to be delivered in succession, separated by $1 / 4$ millisecond from each other, at the repetition rate of 2.5 cycles/second. Since at injection into the FFAG the revolution period is $1.5 \mu \mathrm{s}$, 
a total of about 90 turns need to be stored with the charge-exchange method as it is presently done in the Booster, by letting the negative ions traverse a carbon foil. But now, to optimize the stacking efficiency, we can take advantage of the new ring lattice design, and of the unique features of the FFAG accelerator. The lattice is more compact with stronger focusing that yields smaller lattice functions. The magnet can have an internal gap (required across the injection orbit) somewhat larger than the 3.5 inches of the present Booster. Moreover multi-turn injection can take place during acceleration of the beam pulse, taking advantage of the outward spiraling of the trajectories. We shall describe later a scheme of multi-turn injection for the FFAG. Because of the short period of time the beam spends circulating in the FFAG the entire process of multi-turn injection, acceleration and extraction can be easily simulated on a computer.

\section{FFAG Accelerator Issues}

There are several issues that are peculiar to the mode of operation of a FFAG accelerator:

The same RF cavity system has to provide acceleration simultaneously to different parts of the beam that cross the cavity with different momentum value and at different radial displacement. Thus the width of the RF cavity has to be large enough to accommodate the whole momentum aperture of the beam.

It would be desirable that the beam crosses the cavity (or cavities) always in phase. This requires that the motion is isochronous, that is that any particle takes the same time to go around one revolution. The revolution period in reality varies for two reasons: (a) the particle velocity varies, and (b) the path length varies, from one orbit to the next. In the case of electrons and muons, isochronous conditions can almost be satisfied because the particle velocity does not change very much; but the lattice ought to be carefully designed to cancel as much as possible the variation of the path length with momentum. In the case of protons one has the opposite situation; namely, the variation of the path length has a negligible contribution to the transit time, whereas the variation of the beam velocity is more important. It is then difficult for protons to find ways to fulfill synchronism. This could be achieved only with proper shaping of the magnets longitudinal length. We have abandoned this approach, and simply required that the RF voltage is modulated turn after turn, that is versus momentum, and correspondingly versus the radial position of the beam. This condition is easily satisfied, if at the end of the multi-turn injection, the beam pulse has duration not exceeding the revolution period, and the RF is kept constant.

The most important issue is the search for an ideal lattice that accommodates such a larger momentum aperture that in our case extends from -55 to $+55 \%$. Without entering too much in the detailed discussion of scaling versus non-scaling lattices, it will suffice to say here that it is certainly highly desirable to find a magnet arrangement where the lattice functions, for instance the betatron tunes, do not change too much over the required momentum aperture. It is almost impossible to find a scaling lattice for a 1.5 $\mathrm{GeV}$ proton FFAG, especially if one wants to limit the strength of the bending and focusing fields, that otherwise may require super conducting technology. We had to adopt a non-scaling lattice based on the FDF triplet with modest fields. But we made use of an 
invention, described latter, that minimize the effect of the lattice functions variation with momentum. Essentially, this is achieved with a proper choice of the radial field profile that cancels, at least partially, the variation of the restoring forces with momentum.

Next, we describe the FFAG lattice we have presently derived. It is already a good choice and yet not necessarily optimized. We shall limit our analysis to the acceleration of protons from $200 \mathrm{MeV}$ to $1.5 \mathrm{GeV}$. We are confident that it is then possible to demonstrate how to accelerate heavy ions following essentially the same procedure.

\section{The Lattice of the AGS-FFAG}

At the cost of being too repetitious, we want to stress again that the lattice solution we describe next for the $1.5-\mathrm{GeV}$ FFAG accelerator is a preliminary design. It certainly has all the required features in place, but it is also possible to improve on it for a more optimized solution.

The circumference of the accelerator, that from now we shall simply label as the AGSFFAG, has a circumference of $244.44 \mathrm{~m}$, somewhat larger than the present AGS-Booster, and is made of $\mathrm{N}_{\mathrm{P}}=42$ identical periods, each $5.82 \mathrm{~m}$ long. The period, as shown in Fig. 6 , is made of three sector, combined-function magnets in the symmetric FDF arrangement. The trajectory shown is that of the reference momentum value $\mathrm{p}_{\mathrm{o}}$ taken exactly half way between injection and extraction values as shown in Table 1 . The circumference of the ring quoted above corresponds to the reference (or central) trajectory shown in Fig. 6.

Table 1. Proton Beam Kinematic Parameters

\begin{tabular}{|c|c|c|c|}
\hline & $\underline{\text { Injection }}$ & $\underline{\text { Central }}$ & Extraction \\
\hline Kinetic Energy, MeV & 200 & 786.707 & 1,500 \\
\hline$\beta$ & 0.566163 & 0.83913 & 0.922997 \\
\hline$\gamma$ & 1.21316 & 1.83847 & 2.5987 \\
\hline Momentum, $\mathrm{MeV} / \mathrm{c}$ & 644.441 & $1,447.47$ & $2,250.51$ \\
\hline Magnetic Rigidity, kG-m & 21.496 & 48.2819 & 75.0679 \\
\hline
\end{tabular}



Figure 6. The AGS-FFAG Period (FDF) and the Central-Momentum ( $\mathrm{p}_{\mathrm{o}}$ ) Trajectory. 
To notice that the entrance and exit faces of the D-sector magnet are parallel to neighboring ones of the other two F-sector magnets. Thus, the entrance and exit angles the reference trajectory makes with each magnet are identically equal to zero. This may not be true for an off-momentum trajectory. The lattice parameters are shown in Table 2. The $\mathrm{F}$ and $\mathrm{D}$ magnets are separated by a drift $\mathrm{g}=0.30 \mathrm{~m}$; and a relatively long insertion $2 \mathrm{~S}=1.41 \mathrm{~m}$ separates the FDF periods from each other.

Table 2. Magnet Parameters

\begin{tabular}{lll} 
Magnet Type & \multicolumn{1}{c}{ F } & \multicolumn{1}{c}{ D } \\
Arc Length, m & 0.509444 & 2.79514 \\
Bending Field, kG & -5.29169 & 4.51305 \\
Gradient, kG/m & 33.9174 & -12.4036 \\
Field Index, n & 74.4451 & -37.4293 \\
Bending Radius, m & -10.2943 & 12.0705 \\
Bending Angle, mrad & -49.4877 & 231.569 \\
Sagitta, cm & 0.355537 & 9.12532
\end{tabular}

The values shown in Table 2 again correspond to the reference trajectory. The actual bending (and focusing) field varies across the radial aperture (that is the momentum) as we shall see soon. Notice that whereas the central D-sector magnet bends inward, the two F-sector magnets bend outward. But parameters are adjusted so that the overall bending angle per period is $2 \pi / \mathrm{N}_{\mathrm{p}}$. Thus, since we are assuming circulation of protons (with positive electric charge) in the clockwise direction, the bending field $\mathrm{B}$ and the curvature $1 / \rho$ in the D-sector magnet are positive, and negative in the F-sector magnets. The actual field profile in each of the two types of magnets is shown in Fig. 7 versus the radial ( $\mathrm{x}$, with $\mathrm{x}=0$ corresponding to the reference trajectory) aperture. Injection is located in proximity of $x=-0.30 \mathrm{~m}$ and extraction at $\mathrm{x}=0.10 \mathrm{~m}$. The bending field in the F-sector magnet reverses sign in proximity of the extraction orbit. This can be avoided by readjusting, only locally, the profile. The gradient is negative in the D-sector magnet and positive in the F-sector magnets, as it was required. The field index $\mathrm{n}$ shown in Table 2 corresponds to the field profile in proximity of the central trajectory where it is related to the field gradient $G$ by the relationship $G=n B / \rho$. We shall explain the origin of the field profile and the extension of the radial aperture later.

We have intentionally kept the magnitude of the bending field in proximity of the extraction orbit to reasonably low values, not exceeding $3 \mathrm{kG}$, in the case one desires a continuous acceleration of negative-ions $\mathrm{H}^{-}$, and wants to avoid excessive losses by fieldinduced electron stripping. Though in the case of acceleration of negative ions the direction of motion should be inverted to preserve the polarity of bending and focusing. Moreover, a CW mode of acceleration would require a more sophisticated technique, and probably another invention.

We have first estimated the lattice functions along the length of one period in proximity of the reference trajectory. They are displayed in Fig. 8. The amplitude lattice functions $\beta_{\mathrm{H}}$ and $\beta_{\mathrm{V}}$ have smaller values than those corresponding to the present Booster lattice, 
with obvious improvement on the available dynamical aperture. The dispersion $\eta$ is smaller in the central region of the D-sector magnet, and larger in the long-drift $\mathrm{S}$. The lattice parameters are summarized in Table 3.

The lattice has the capability to accept a momentum variation around the central value of $\pm 55.5 \%$. We have opted for the FDF triplet arrangement, instead of the opposite DFD, because it allows a considerable smaller dispersion and, henceforth, a more manageable radial aperture of the magnets.
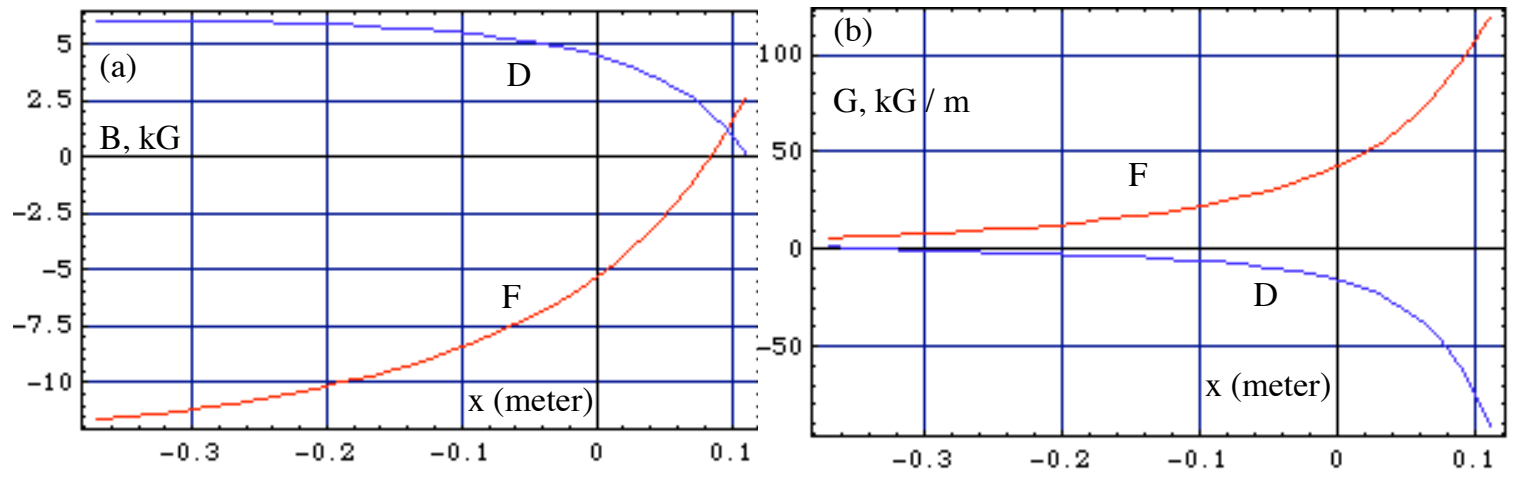

Figure 7. (a) Bending Field B and (b) Gradient G Profiles versus radial aperture. Central Trajectory corresponds to $\mathrm{x}=0$



Figure 8. The AGS-FFAG Lattice Functions for the Central Orbit $(\mathrm{x}=0)$

\section{Momentum Dependence of the AGS-FFAG Lattice}

At the beginning of our study we found very difficult to find a lattice solution stable over the required momentum aperture with a conventional field profile described by a constant field index $\mathrm{n}$. We opted then for a linear profile with constant gradient $\mathrm{G}$ across the aperture. We were eventually able to find stable solutions; but, since we were adopting what some experts in the field call a non-scaling lattice, we were initially frustrated by 
the too large variations of the lattice functions from injection to extraction that, in our view, would have made the operation of the accelerator difficult and impractical. We begin then looking for ways to optimize the field profile to yield the smaller variation of the lattice functions as possible. We went back, for that, to the basic equations of motion.

Table 3. The AGS-FFAG Parameters for the Reference Trajectory

Circumference
Number of Periods
Period Length
Period Structure
Short Drift, g
Long Drift, $\mathrm{S}$
$\beta_{\mathrm{H}} \max ($ in $\mathrm{S}$ )
$\beta_{\mathrm{V}} \max ($ in $\mathrm{D})$
$\eta$ max (in S)
Phase Advance / Period, H/V
Betatron Tunes, H/V
Natural Chromaticity, H/V
Momentum Compaction Factor
Transition Energy, $\gamma_{\mathrm{T}}$

$244.439 \mathrm{~m}$
42
$5.81998 \mathrm{~m}$
$\mathrm{~S}$ F g D g F $\mathrm{S}$
$0.30 \mathrm{~m}$
$1.405954 \mathrm{~m}$
$7.2731 \mathrm{~m}$
$9.03163 \mathrm{~m}$
$0.2674 \mathrm{~m}$
$97.7143^{\circ} / 97.7143^{\circ}$
$11.40 / 11.40$
$-1.21304 /-1.31337$
$-6.3266 \times 10^{-4}$
$39.7572 \mathrm{i}$

The following equations of motion are derived in the Appendix in the case a particle with electric charge $q$ and momentum $\mathrm{p}$ moves in a constant magnetic field with gradient:

$\begin{array}{llll}\mathrm{x}^{\prime \prime}+(\mathrm{hB}+\mathrm{G}) \mathrm{qx} / \mathrm{cp} & =\mathrm{h}\left(1-\mathrm{p}_{0} / \mathrm{p}\right) \\ \mathrm{y}^{\prime \prime}-\mathrm{qGy} / \mathrm{cp} & = & 0\end{array}$

Observe the presence of the curvature term (h) in the radial (x) equation of motion but not in the vertical one, Moreover, observe the dependence with the actual momentum $p$ of the particle. As it is customary in this situation, let us introduce the relative momentum deviation $\delta$, so that $\mathrm{p}=\mathrm{p}_{0}(1+\delta)$. The momentum dependence does not enter in the curvature function $\mathrm{h}(\mathrm{s})$ that has a pure geometric definition, but it enters as the denominator of the field components. Our idea is to offset the momentum dependence allowing the field index $\mathrm{n}$, describing the field profile, to absorb it. Since ultimately $\mathrm{x}=$ $x(\delta)$ and $n=n(x)$, we can set the relation between $n$ and $\delta$, for both sector magnets, as follows

$\mathrm{n}=\mathrm{n}_{\mathrm{F}, \mathrm{D}}(1+\delta)$

where $n_{F, D}$ are the values shown in Table 2 that correspond to the central trajectory. Other similar relations can of course also be used. It is important that eventually the variation of the field $\mathrm{n}$ with $\mathrm{x}$ is consistent with Maxwell's equations (at least up to quadratic order terms). We have left the momentum dependence on the curvature term at the right hand side of Eq. (1) unchanged. That is the source of dispersion. 
Thus our approach is clear: with relation (3) inserted in the equations of motion, the momentum dependence is, at least partially, offset. We have developed a SYNCH-like code (using MATHEMATICA [12]) where the symbolism represented by Eq. (3) is included. First, we derive the fixed closed orbits for different momentum values in the range $\delta= \pm 0.60$. The bundle of trajectories for our selected lattice is shown in Fig. 9. The radial extension is reasonable confined; it is $35 \mathrm{~cm}$ in the middle of the long-straight $\mathrm{S}$ and only $10 \mathrm{~cm}$ in the center of the D-sector magnet. The result depends on our choice to have limited the magnitude of the bending field. Fig. 10 is the plot of the dispersion $\eta$ for the same momentum values, and Fig.s 11 and 12 are the plots of the amplitude lattice functions $\beta_{\mathrm{H}}$ and $\beta_{\mathrm{v}}$. Though, as expected, the variation of the dispersion with momentum is large, that of $\beta_{\mathrm{H}}$ and $\beta_{\mathrm{V}}$ is very compact: a satisfactory result! The betatron tunes $v_{\mathrm{H}}$ and $v_{\mathrm{V}}$ are plotted together in Fig. 13. Notice the flatness of the vertical tune, whereas there is still an appreciable variation of the horizontal tune versus momentum (or position). That is caused by our choice of the $n(\delta)$ dependence in Eq. (3) and due to the curvature term. In any case the variation is less than a unit. Thus crossing of integral resonances can be avoided, and we are left with the crossing of a half-integral resonance that is not systematic, that is it is not intrinsic to the lattice periodicity. The hope is that with a better choice of the $n(\delta)$ dependence one can reach a compromise between horizontal and vertical components of motion that can reduce the range of the tune variation even farther. With the use of the powerful symbolic language of MATHEMATICA, it is possible to invert relationships and to derive the actual field profile versus radial aperture as the one shown in Fig. 7.

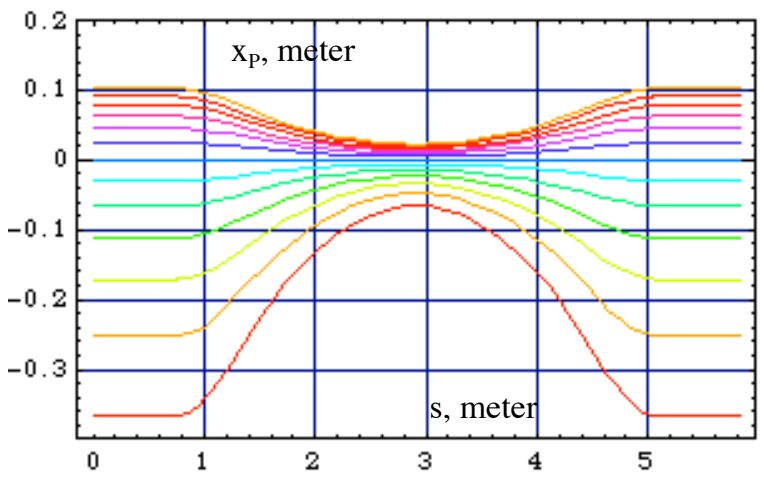

Figure 9. Fixed Orbits vs. Period path length $\delta= \pm 0.6$ in steps of 0.1

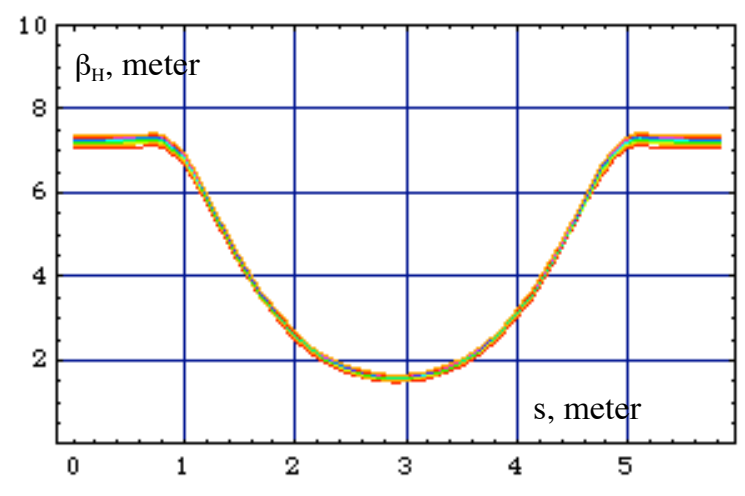

Figure 11. $\beta_{\mathrm{H}}$ vs. Period path length $\delta= \pm 0.6$ in steps of 0.1

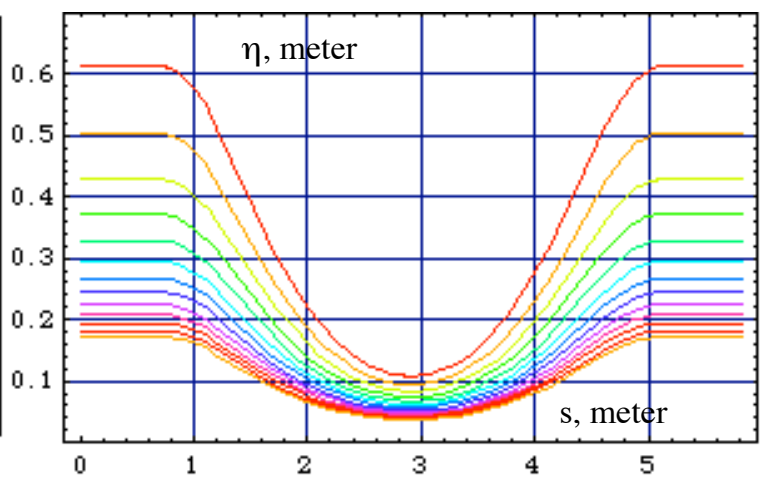

Figure 10. Dispersion Funct. vs. Period path length $\delta= \pm 0.6$ in steps of 0.1

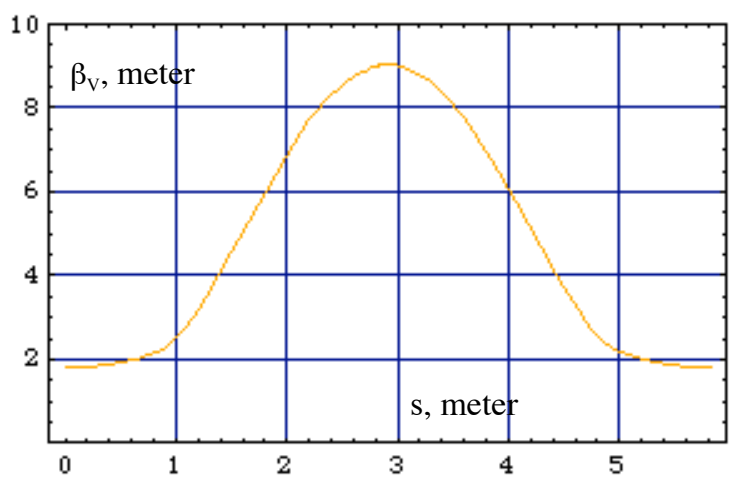

Figure 12. $\beta_{\mathrm{v}}$ vs. Period path length $\delta= \pm 0.6$ in steps of 0.1 

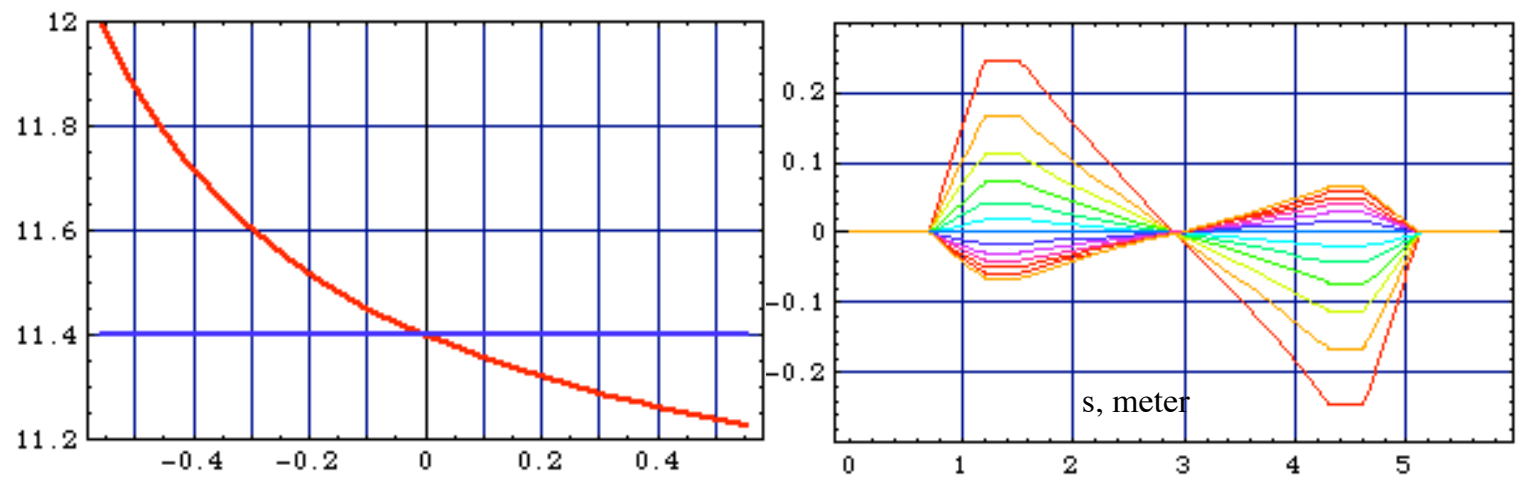

Figure 13. Betatron Tunes $v_{\mathrm{H}}$ and $v_{\mathrm{V}}$ vs. Momentum Aperture Figure 14. Trajectory Angle $\mathrm{x}_{\mathrm{P}}{ }^{\prime}$ for $\delta= \pm 0.6$ in steps of 0.1

Fig. 14 gives the derivative $\mathrm{x}_{\mathrm{P}}{ }^{\prime}$ of the fixed closed orbit radial location $\mathrm{x}_{\mathrm{P}}$ (shown in Fig. 9) versus the curvilinear coordinate $s$. The derivative $x_{P}{ }^{\prime}$, estimated at the proper locations, gives in turn the entrance and exit angles the off-momentum trajectories make with each of the sector magnets. As we can see from Fig.14, these angles can be as large as $250 \mathrm{mrad}$. They have a focusing/defocusing effect, and their contribution to the lattice functions has been properly included in our calculation.

\section{Magnet Considerations}

The field profile in the two sector magnets is shown in Fig. 7. The bending field has the largest magnitude on the injection orbit and decreases steadily toward the extraction orbit, in both magnets, except that the polarity is reversed: it is positive in the D-sector magnet, and negative in the F-sector magnet. Programs like TOSCA or OPERA can eventually be used to design magnets with the proper iron shape to create the required profile. For the moment it will suffice to notice that the magnet crosssection will look like that of Fig. 15. Since the beam will spend a very short period of time circulating in the ring, only a modest vacuum pressure is required and there may be thus no need of an internal vacuum chamber. Moreover, because the magnetic field is constant, there are no eddy-currents and if a vacuum chamber is desired, it can be made of solid metallic material of any thickness. The field is

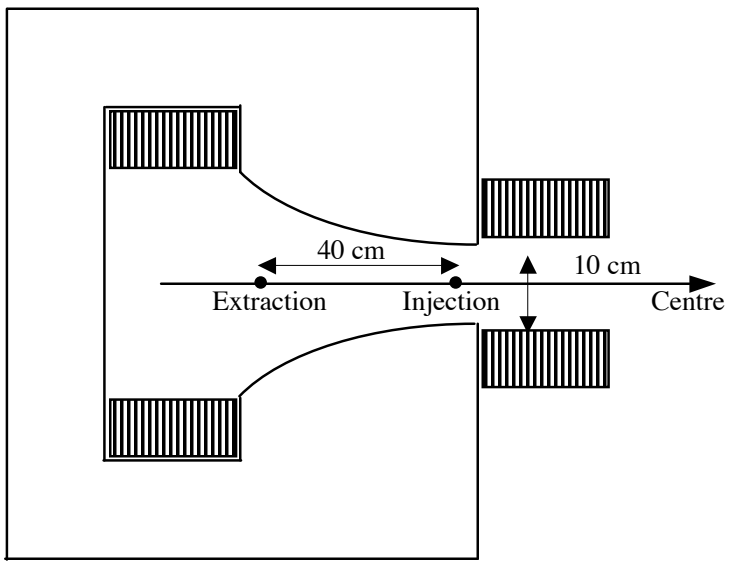

Figure 15. Cross-Section of the F-sector Magnet constant in time and the magnet iron can be made of solid blocks without lamination. It will be curved to follow closely the curvilinear trajectory. The internal width is about 40 $\mathrm{cm}$, between injection and extraction orbit, including also space for the contribution of the betatron emittance to the beam size. The vertical gap is about $10 \mathrm{~cm}$. These dimensions apply to both the D and the F-sector magnet. 


\section{Injection of Protons into the AGS-FFAG}

About a hundred turns of negative-ions $\mathrm{H}^{-}$are to be injected from the 200-MeV Drift Tube Linac into the AGS-FFAG. The beam will come from an elevation above the FFAG magnets, and then deflected downward on the FFAG mid-plane with two vertical dogleg magnets A1 and A2 as shown in Fig. 16. Quadrupoles are also inserted along the injection transport line for matching of the lattice functions to values corresponding to injection into the FFAG ring. Prior to the start of injection, a horizontal orbit bump of the injection orbit is created. The bump spans over two periods, and is created by two bump magnets B1 and B2 separated ideally by a phase advance of $180^{\circ}$. At the center of the bump, at about the middle of the 1.4-m long drift space, there are two horizontal dogleg magnets $\mathrm{C} 1$ and $\mathrm{C} 2$ separated enough for the insertion of a Carbon foil that, traversed by the beam, will cause stripping. The beam rms spot size on the foil is $3 \mathrm{~mm}(\mathrm{H}) \times 1 \mathrm{~mm}$ $(\mathrm{V})$. The beam is then captured on the injection orbit by the charge-exchange process. Multi-turn injection last about $143 \mu \mathrm{s}$. At the end of the process the beam should extend over the length of one turn, with a gap of about $300 \mathrm{~ns}$, and then accelerated.

During injection the accelerating RF is on, set to zero phase angle and constant voltage. The RF is $201.25 \mathrm{MHz}$ and matches that of the DTL. The proton beam at injection is also bunched at the same frequency. The harmonic number is $h=290$. The Linac bunches are transferred to the RF buckets where they are stacked turn-after-turn during the multi-turn injection. About a hundred Linac bunches will fall in a single RF bucket. The beam is pre-chopped at the beginning of the DTL for the duration of about $80 \%$ of the revolution period. The chopping frequency equals the injection revolution frequency. This will create a gap in the beam of about 300 ns that will make easier the extraction process. Table 4 gives the summary of the beam parameters at injection.

Table 4. Beam Parameters at Injection into the FFAG

$\begin{array}{ll}\text { Linac Peak Current } & 35 \mathrm{~mA} \\ \text { Revolution Period } & 1.44 \mu \mathrm{s} \\ \text { No. of Protons / FFAG pulse } & 2.5 \times 10^{13} \\ \text { Chopping Ratio } & 0.80 \\ \text { Chopping Frequency } & 0.694 \mathrm{MHz} \\ \text { Single Pulse Length } & 143 \mu \mathrm{s} \\ \text { No. of Turns Injected / pulse } & 100 \\ \text { Beam Emittance, rms norm. } & 1 \pi \mathrm{mm}-\mathrm{mrad} \\ \text { Bunching Frequency } & 201.25 \mathrm{MHz}\end{array}$

\section{Acceleration of Protons in the AGS-FFAG}

It desirable to preserve the $201.25 \mathrm{MHz}$ bunching of the beam as it comes out of the DTL and is injected into the AGS-FFAG. It is also desirable to shorten the Linac cycle time as much as possible, for which we need a fast accelerating cycle in the FFAG. We thus assume that the accelerating RF system at injection operates also at the same frequency of 201.25 MHz. At injection that corresponds to harmonic number $\mathrm{h}=290$. 


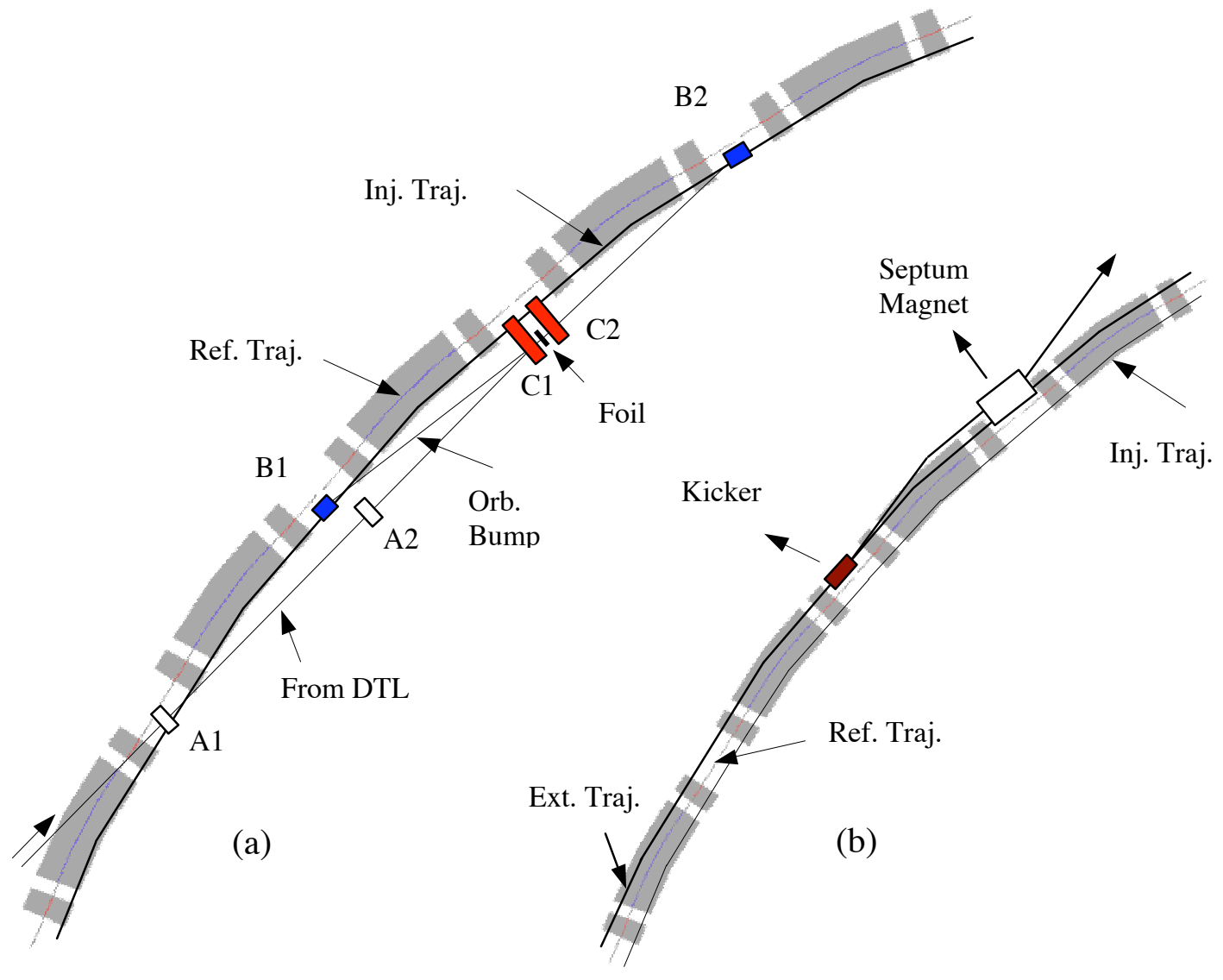

Figure 16. (a) Injection of $\mathrm{H}^{-}$into the AGS-FFAG. (b) Single-Turn Extraction of Protons

Because the beam velocity changes, the RF should be modulated over the acceleration period that we take here to be only of a hundred revolutions. The frequency modulation is though difficult, if not impossible, to achieve in such a short period of time at such large RF. We opted thus for a constant RF during the entire acceleration cycle, but allowed a programmed RF voltage modulation to compensate for the variation of the beam velocity. For that we require that at the end of a revolution the beam receives an energy gain $\mathrm{eV}$ such that the revolution period decreases, between two consecutive crossings of the RF cavity system, by an amount exactly equal to the RF period (harmonic jump), according to the following equation

$\mathrm{eV}=\mathrm{E}_{0} \beta^{2} \gamma^{3} / \mathrm{h}\left(1-\alpha_{\mathrm{p}} \gamma^{2}\right)$

where $E_{0}$ is the rest energy, $\beta$ and $\gamma$ the usual velocity and energy relativistic factors, $\alpha_{p}$ the momentum compaction factor (that for our lattice is negative), and $\mathrm{h}$ the harmonic number that obviously decreases by one unit every revolution as shown in Fig. 17. The actual variation of the path length per period versus the relative momentum deviation is given in Fig. 18. The variation gives only a small contribution to the transit time across a period, as that is mostly determined by the variation of the beam velocity. The RF voltage V program is shown in Fig. 19. 


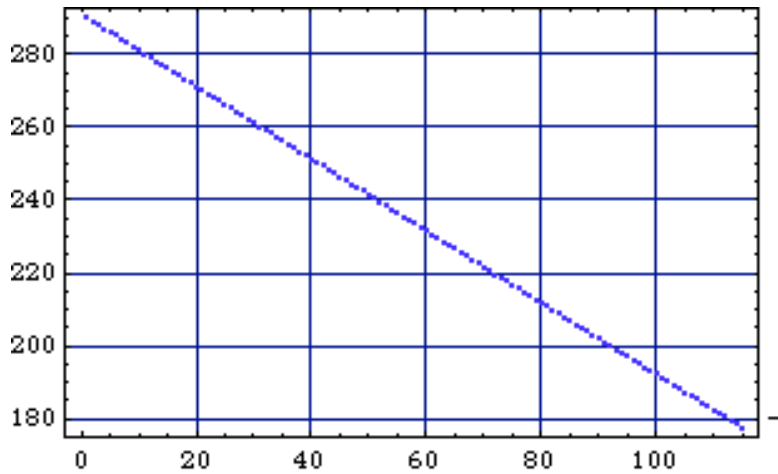

Figure 17. RF Harmonic Number h vs. number of turns



Figure 19. RF Voltage vs. number of turns

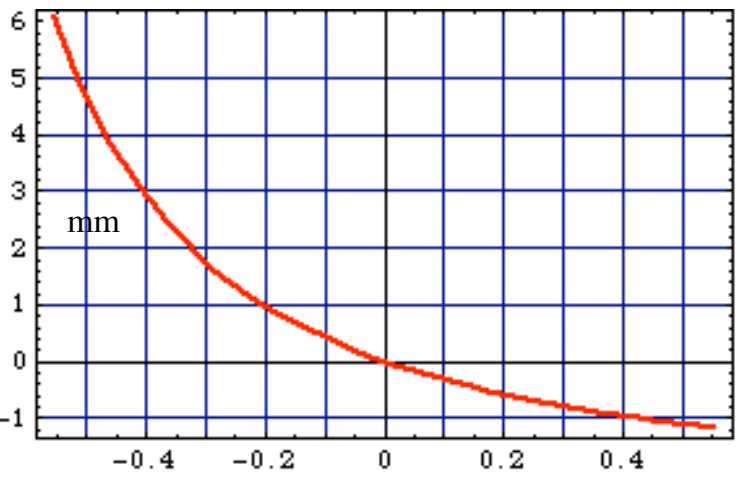

Figure 18. Period Length Variation vs. $\delta$

It takes 114 revolutions and $132 \mu$ s for the entire acceleration cycle from $200 \mathrm{MeV}$ to $1.5 \mathrm{GeV}$. During the cycle the RF voltage varies between 2 and $70 \mathrm{MV}$. Table 5 gives the summary of the RF parameters during acceleration. Because of the relative small length of the beam bunches, and the short duration of the acceleration cycle, each bunch is taken as a point on top of the RF waveform crest, that is a RF phase of $90^{\circ}$. The required voltage is

achieved with any number of cavities as dictated by RF design considerations. Cavities are of course located in the 1.4-m long straight sections $(\mathrm{S})$. Their radial aperture should be about $40 \mathrm{~cm}$ to allow acceleration over the whole momentum aperture. Voltage will vary as the beam bunches move from one orbit to the next. For this purpose an amplitude and phase loop that locks on the bunch location is also required. The longitudinal dynamics in the AGS-FFAG is simple, and it does not represent a major issue.

Table 5. Acceleration Parameters (Protons) in the AGS-FFAG

Energy Range

Circumference

Harmonic Number

RF

Energy Gain / turn

RF phase angle

Number of Revolutions

Acceleration Period

No. Protons / cycle

FFAG Cycle Period

No. of Pulses / AGS Cycle

DTL Pulse Length
$200-1,500 \mathrm{MeV}$

$244.439 \mathrm{~m}$

$290-176$

$201.25 \mathrm{MHz}$

2-70 MVolt

$\sim 90^{\circ}$

114

$132 \mu \mathrm{s}$

$2.5 \times 10^{13}$

$275 \mu \mathrm{s}$

4

$1.1 \mathrm{~ms}$ 
The revolution frequency is essentially determined by the velocity of the beam. The path length variation with momentum is entirely negligible, as shown in Fig. 18. The momentum compaction factor is negative, yielding imaginary transition energy. This is a fortuitous result due to the choice of the parameters of the lattice geometry, and, mostly, to the inversion of the bending in the magnet triplet. Fig. 20 is a plot of the betatron tunes versus the number of turns during acceleration. Fig. 21 gives the largest horizontal and vertical (rms) beam transverse dimensions. Fig. 22 is a plot of the radial location of the orbit as it is displaced during acceleration. Fig.s 23 and 24 are the plots of the bending fields $\mathrm{B}$ and the gradients $\mathrm{G}$ during the acceleration cycle.



Figure 20. Betatron Tunes vs. number of turns

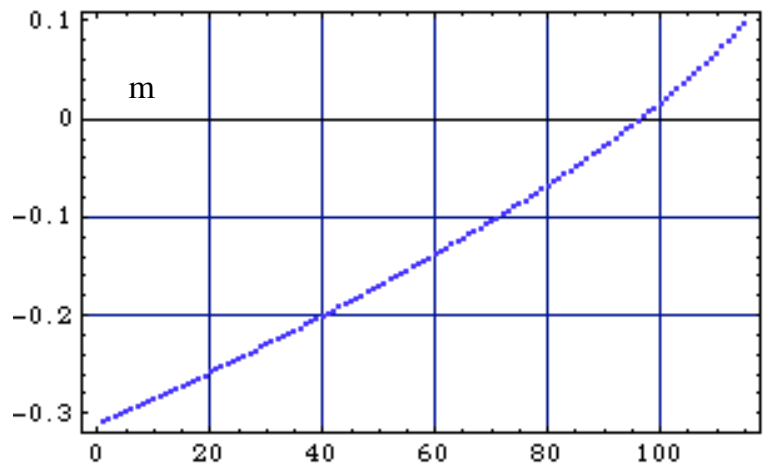

Figure 22. x-Position of the Orbit vs. number of turns

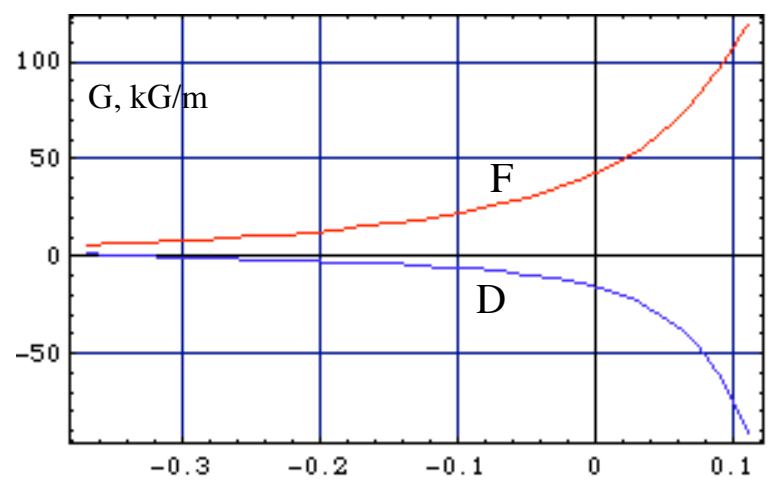

Figure 24. Gradient vs. number of turns

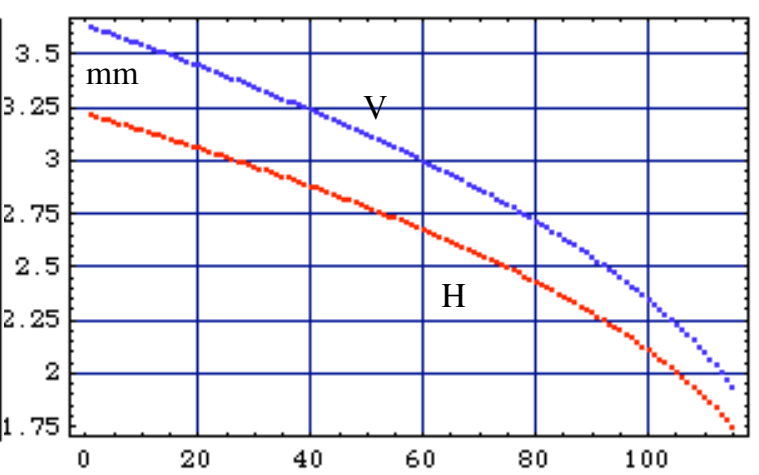

Figure 21. rms Beam Size vs. number of turns

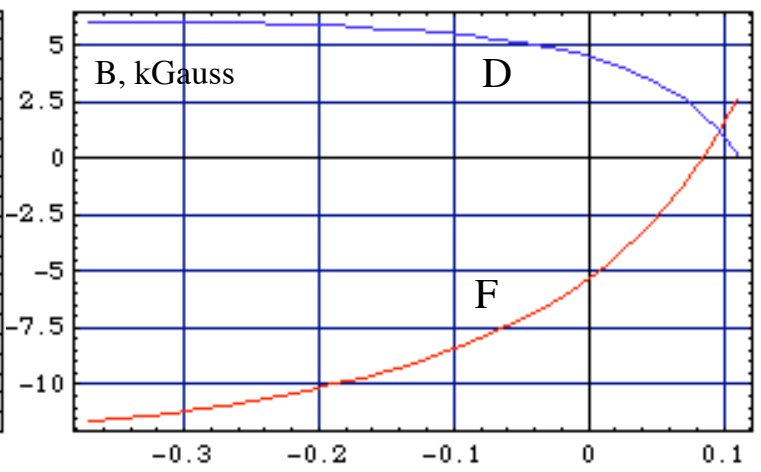

Figure 23. Bending Field vs. number of turns

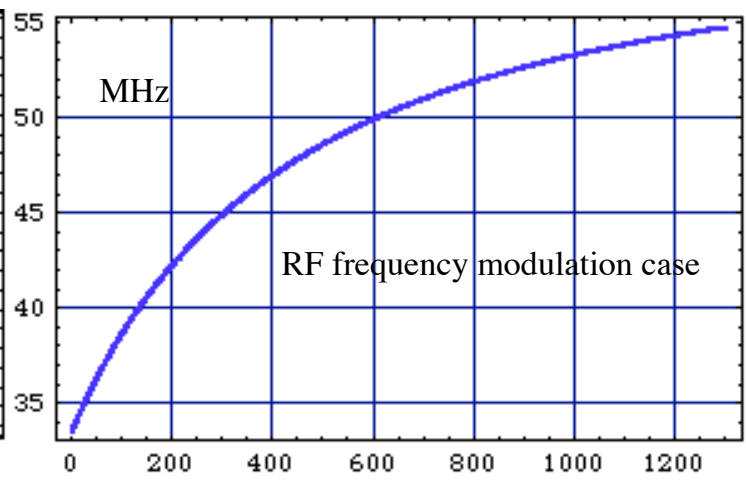

Figure 25. RF vs. number of turns 


\section{Acceleration in a Green-Field 1.5-GeV FFAG}

In the previous section we have described acceleration in the AGS-FFAG. The major constrain was to reduce the DTL cycle period to less than a millisecond because of the present thermal and RF limitations that can be overcome only with major and expensive modifications. Because of that, we have opted for a RF voltage modulation and constant frequency, by letting the harmonic number skip one unit every turn.

But in the case the 1.5-GeV FFAG is to be built on an independent site with a more suitable linac injector, this could be designed beforehand with the capability to operate over longer periods of time. We would then opt for the frequency modulation as the beam is accelerated, preserving the constancy of the harmonic number and of the RF voltage. The example shown here assumes a constant energy gain of $1 \mathrm{MeV} / \mathrm{turn}$ and the harmonic number $\mathrm{h}=48$. Acceleration from $200 \mathrm{MeV}$ to $1.5 \mathrm{GeV}$ takes 1,300 revolutions and $1.31 \mathrm{~ms}$. The required RF swing is shown in Fig. 25. The RF at injection is precisely $1 / 6$ of $201.25 \mathrm{MHz}$ that we still assume to be the bunching frequency of the beam from the injector. If the FFAG were still to fill an accelerator like AGS with four consecutive pulses, it would take about $6 \mathrm{~ms}$. The choice of a considerably higher RF would also consequently make easier the acceleration program in the AGS-like subsequent accelerator.

\section{Extraction from the FFAG}

It is simply required to extract the whole beam in a single turn. At the moment of extraction the revolution period is $0.9 \mu$ s with a beam gap of about $300 \mathrm{~ns}$. The extraction scheme is shown in Fig. 16b. Just prior to extraction, a 1-kG kicker magnet is rapidly turned on in correspondence of the beam gap. The beam is kicked outward and enters a Septum Magnet located upstream of the next 1.4-m long straight where it is initially displaced outward by about $10 \mathrm{~cm}$. This operation will repeat 4 times in succession at 260 $\mu$ s time interval, and at the repetition rate of $2.5 \mathrm{~Hz}$, for the filling of the AGS. Thus the kicker magnet has a rise-time of about $300 \mathrm{~ns}$, or less. The kicker field remains constant for the duration of the beam pulse (about $0.6 \mu \mathrm{s}$ ), and it is finally reset to zero-value in about $250 \mu \mathrm{s}$.

\section{References}

[1] K.R. Symon et al., Phys. Rev. 103, 1837 (1956). Also, T. Ohhawa (1958). Rev. Sci. Instr. 29, 108.

[2] The MURA Staff. Proc. Intern. Conf. On High Energy Accelerators, Brookhaven, 1961. USAEC, Washington, 1961, page 57 and 344.

[3] See for instance, Proc. Of the Workshop on Accelerators for Future Spallation Neutron Sources, Vol. II B, Feb. 16-20, 1993. Picacho Plaza, Santa Fe, NM. K. Ziegler p. 1252, and B. Kustom p. 1264.

[4] S. Machida et al., "Status of the $150 \mathrm{MeV}$ FFAG Synchrotron", PAC 2003, Portland, Oregon, May 12-16, 2003. 
[5] See for instance, A. G. Ruggiero, "Design Considerations on a Proton Superconducting Linac", BNL 62312 (Informal Report), Aug. 1995. BNL Interdepartmental Study of a Spallation Neutron Source.

[6] D. Trbojevic et al., "Electron Acceleration for e-RHIC with Non-scaling FFAG", Contribution to EPAC 2004, July 5-9, Lucerne, Switzerland.

[7] D. Trbojevic et al., "FFAG Lattice for Mun Acceleration with Distributed RF", PAC 2003, Portland, Oregon, May 12-16, 2003.

[8] AGS Super Neutrino Beam Facility, (NWG Report-II), Editor D. Raparia. BNL 71228 (Informal Report), April 2003.

[9] A.G. Ruggiero et al., "Design of a 1.2-GeV SCL as New Injector for the BNL AGS", BNL 71706-2003-CP. Submitted to SRF 2003 Workshop, Travemunde (Lubeck), Germany. Sept. 8-12, 2003.

[10] The SNS Projec: http://www.sns.gov

[11] I. Marneris et al., "Proposed Upgrade of the AGS MMPS to 2.5 up to $5.0 \mathrm{~Hz}$ ". Contribution to EPAC 2004, July 5-9, Lucerne, Switzerland.

[12] MATHEMATICA, Wolfram Research, http://www.wolfram.com

[13] E.D. Courant and H.S. Snyder, Annals of Physics: 3, 1-48 (1958).

\section{Appendix}

It is well known [13] that the equations of motion of a particle with electric charge $q$ and total momentum p, moving in a region of space where only magnetic fields are present, can be derived from the Hamiltonian (valid for planar motion)

$\mathrm{H}=-\mathrm{qA}_{\mathrm{s}} / \mathrm{c}-(1+\mathrm{hx})\left[\mathrm{p}^{2}-\left(\mathrm{p}_{\mathrm{x}}-\mathrm{qA} \mathrm{A}_{\mathrm{x}} / \mathrm{c}\right)^{2}-\left(\mathrm{p}_{\mathrm{y}}-\mathrm{qA} \mathrm{A}_{\mathrm{y}} / \mathrm{c}\right)^{2}\right]^{1 / 2}$

where we have adopted a curvilinear coordinate system described by the geometrical curvature function $h=h(s)$. Expanding the square root, and retaining only up to quadratic terms, the Hamiltonian simplifies:

$\mathrm{H}=-\mathrm{qA}_{\mathrm{s}} / \mathrm{c}-\mathrm{phx}+\left(\mathrm{p}_{\mathrm{x}}^{2}+\mathrm{p}_{\mathrm{y}}^{2}\right) / 2 \mathrm{p}$

and we have assumed that the vector potential components $A_{x}=A_{y}=0$. We derive then the equations of motion

$$
\begin{aligned}
& \mathrm{x}^{\prime \prime}=(\mathrm{q} / \mathrm{pc}) \partial \mathrm{A}_{\mathrm{s}} / \partial \mathrm{x}+\mathrm{h} \\
& \mathrm{y}^{\prime \prime}=(\mathrm{q} / \mathrm{pc}) \partial \mathrm{A}_{\mathrm{s}} / \partial \mathrm{y}
\end{aligned}
$$

Again if we retain only quadratic order terms, denoting with B the bending field and $G$ the gradient,

$$
\mathrm{A}_{\mathrm{s}}=-\mathrm{Bx}-(\mathrm{hB} / 2+\mathrm{G} / 2) \mathrm{x}^{2}+(\mathrm{G} / 2) \mathrm{y}^{2}
$$


Insertion of Eq. (A5) in Eq.s (A3 and A4) finally gives Eq.s (1 and 2) in the main text, if we also set

$\mathrm{qB} / \mathrm{ch} \quad=\quad \mathrm{p}_{0}$ 